\title{
Comparative Analysis of the DFB Fiber Laser and Fiber-Optic Interferometric Strain Sensors
}

\author{
G.A.Cranch $^{\mathrm{b}, \mathbf{a}^{*}}$, G.M.H.Flockhart ${ }^{\mathrm{b}, \mathrm{a}}$, C.K.Kirkendall ${ }^{\mathrm{b}}$ \\ ${ }^{\mathrm{b}}$ Naval Research Laboratory, Code 5674, Washington DC 20375, \\ ${ }^{\mathrm{a}}$ SFA Inc, Crofton, MD 21114
}

\begin{abstract}
The DFB fiber laser strain sensor is shown to provide an improvement in the minimum detectable length change by a factor of 275 at $2 \mathrm{kHz}$ for an applied strain when compared with an interferometric sensor interrogated by an equivalent DFB laser. This corresponds to a strain resolution enhancement by a factor of 18 , if the strain is applied over a $10 \mathrm{~cm}$ length of fiber. The ability of the fiber laser sensor to operate in its thermodynamic noise limit is shown to be the primary reason for this enhanced sensitivity. Thermodynamically limited noise performance is demonstrated with three interrogation methods.
\end{abstract}

\section{INTRODUCTION}

The distributed feedback (DFB) fiber laser provides new possibilities for developing very high performance ${ }^{\mathrm{I}}$, small size, strain sensors. Probably the most successful, existing high-performance fiber-optic strain sensors are based on fiberoptic interferometry. Indeed, the development of the fiber laser operating in the $1550 \mathrm{~nm}$ erbium window has permitted very high performance to be obtained from interferometric sensors interrogated by fiber laser sources [1] costing considerably less than existing high-performance lasers, such at the diode-pumped Nd:YAG non-planar ring laser [2]. In many types of interferometric sensor, the phase resolution is determined by laser frequency induced phase noise, which is generated in unbalanced interferometers. Balancing the optical paths thus reduces this noise source. However, in many interrogation schemes a non-zero path imbalance will exist due either to engineering constraints or the requirement of a small path imbalance in order to implement an interrogation scheme. The fiber laser sensor (FLS) offers an interesting alternative configuration to the interferometric sensor, and is implemented by simply exchanging the functions of the interferometer and laser in an interferometric sensor. In the FLS, the laser now becomes the strain sensor, where the applied strain modulates the wavelength or frequency of the laser, and the interferometer acts to decode the frequency shift of the laser. It will be shown that this configuration provides a higher sensitivity per unit length than the interferometric sensor. Thus, for any strain sensing configuration where the interaction length is limited, the FLS may either provide a performance benefit or permit equivalent sensitivity to be achieved in a smaller size transducer. Although a seemingly straightforward and beneficial exchange, the performance of the FLS is critically dependent on the laser output spectral properties. When used as a laser source, it can generally be ensured that the environmental operating conditions are carefully controlled. However, when configured as a sensor, the operating conditions are much less certain, and the laser must therefore by designed to tolerate more variation in operating conditions without significant effect on the spectral properties. What has allowed this concept to be practically useful is the significant developments that have taken place in fiber Bragg grating lasers over the last $\sim 15$ years. High performance strain measurement is achieved when the fiber laser operates in a single longitudinal mode, which requires a very short laser cavity. Considerable improvements in laser stability were obtained by the implementation of the distributed feedback fiber laser [3]. This laser configuration consists of a single Bragg grating, a few $\mathrm{cm}$ in length, with a phase shift imposed to force single frequency operation. The lasing mode is now effectively locked to the resonance caused by the phase shift. This structure provides considerable resilience to environmental perturbations such as temperature gradients and bending of the fiber. These developments have yielded realistic demonstrations of sensors based on the DFB fiber laser [4-6].

The aim of the manuscript is to compare the performance of the fully optimized interferometric sensor interrogated by the DFB fiber laser with the DFB fiber laser sensor. The performance benefits of the FLS are calculated and a discussion

\footnotetext{
*Email: geoff.cranch@nrl.navy.mil

${ }^{\mathrm{I}}$ High-performance is a relative term and in the present context refers to strain sensors capable of achieving strain resolutions approximately in the 1 to $100 \mathrm{f} \varepsilon$ range. For comparison, standard fiber Bragg grating strain sensors achieve $\sim 1 \mu \varepsilon$ resolution (dc), interferometrically interrogated FBGs achieve

$\sim 1 \mathrm{n} \varepsilon$ resolution (ac) and interferometric sensors achieve sub p $\varepsilon$ resolution (ac). Note that dc generally refers to frequencies less than $\sim 1 \mathrm{~Hz}$.
}

Third European Workshop on Optical Fibre Sensors, Antonello Cutolo, Brian Culshaw, José Miguel López-Higuera, Eds., Proceedings of SPIE Vol. 6619, 66192C, (2007) · 0277-786X/07/\$18 - doi: 10.1117/12.738561 
on efficient implementations of the wavelength decoding interferometer based on (i) active-homodyne, (ii) phasegenerated carrier and (iii) the $3 \times 3$ interferometer is given.

\section{STRAIN SENSORS}

The output spectral properties of the fiber laser strongly influence the performance of both the interferometric sensor and the FLS. Two important properties are the relative intensity noise and frequency noise, from which the performance of most strain sensor configurations can be predicted. Two DFB fiber lasers, which exhibit similar output properties and referred to as FL1 and 2, are used in this work. The DFB fiber laser under test is pumped at $\sim 980 \mathrm{~nm}$ with a grating stabilized pump diode producing up to $200 \mathrm{~mW}$. Both FLs exhibit single frequency and polarization mode output. The relative intensity noise (RIN) of FL1 is shown in fig. 1(i). This exhibits a strong relaxation oscillation at $210 \mathrm{kHz}$, which increases the RIN to $-82 \mathrm{~dB} / \mathrm{Hz}$. Between $100 \mathrm{~Hz}$ and $100 \mathrm{kHz}$, the FL RIN is close to the pump RIN. In the case of the FLS, a significant source of excess noise due to RIN is that caused by noise aliasing, when the detected power is not sufficiently low-pass filtered to remove the relaxation oscillation peak.
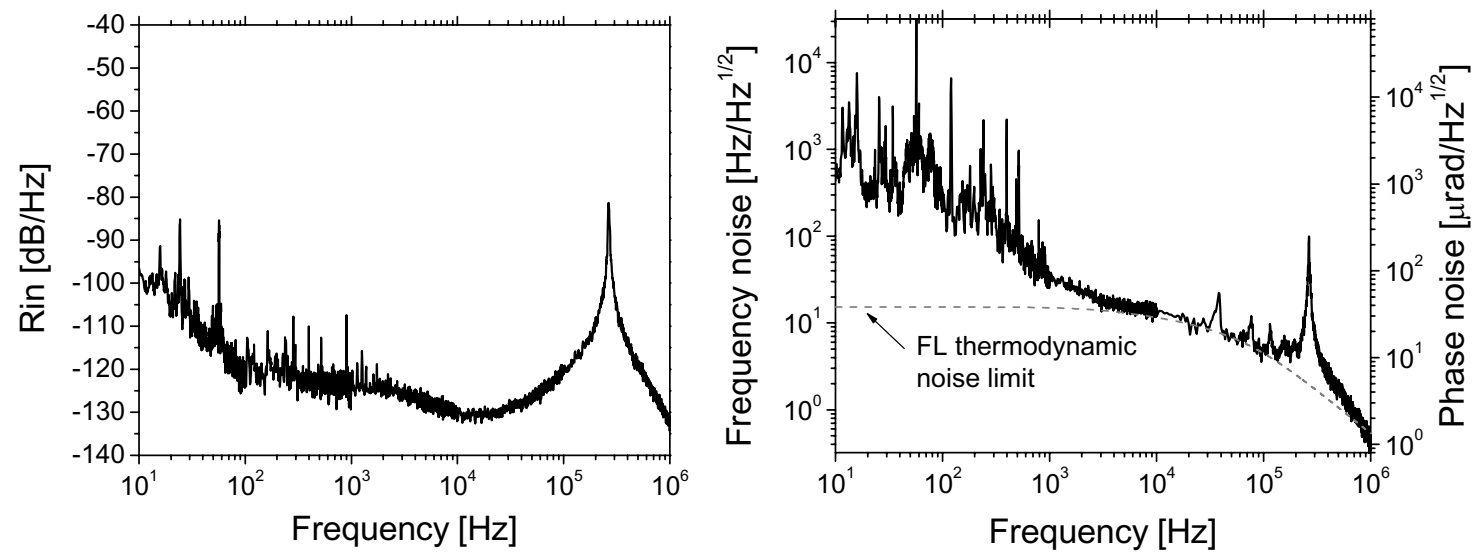

Figure 1: DFB fiber laser (i) relative intensity noise and (ii) frequency/phase noise (80m imbalance)

The limiting noise source in the FLS is shown to be frequency noise, which exhibits several features. The frequency noise of the laser is measured by injecting the laser output into a fiber-optic Mach-Zehnder interferometer with an $80 \mathrm{~m}$ fiber path imbalance and converting the interferometric phase noise into input frequency noise. This is shown in fig. 1(ii) along with the equivalent phase noise. Below $1 \mathrm{kHz}$ the frequency noise is strongly influenced by environmental acoustical and vibration noise. Between $\sim 3 \mathrm{kHz}$ and $100 \mathrm{kHz}$, the noise is limited by thermodynamic noise, first noted in [7] (spontaneous emission noise is estimated to be over an order of magnitude lower than this noise source). The theoretical thermodynamic noise, described in [8], is also plotted, for an effective cavity length of $6.5 \mathrm{~mm}$, yielding a frequency noise of $14.5 \mathrm{~Hz} / \mathrm{Hz}^{1 / 2}$ or phase noise of $33 \mu \mathrm{rad} / \mathrm{Hz}^{1 / 2}$ at $2 \mathrm{kHz}$. For comparison, the phase noise due to thermodynamic noise in the measurement interferometer is $0.238 \mu \mathrm{rad} / \mathrm{Hz}^{1 / 2}$ (a factor of $\sim 252$ less than the measured phase noise@ $2 \mathrm{kHz}$ ). It is worth noting that although the thermodynamically induced phase noise in the interferometer is much lower, this noise floor in a practical interferometric sensor is never realized (unless the fiber lengths are $>1 \mathrm{~km}$ ), since other noise sources, typically detector or laser noise limit the phase resolution to greater than a microradian. Above $100 \mathrm{kHz}$, an increase in noise due to the relaxation oscillation peak is observed. Note that the smaller peaks in the frequency noise spectrum over the range $10 \mathrm{kHz}-100 \mathrm{kHz}$ are thought to originate from the mounting.

\section{FIBER-OPTIC INTERFEROMETRIC STRAIN SENSOR}

The interferometric sensor configuration is shown in fig. 2 and utilizes the frequency-modulated phase-generated carrier (FM-PGC) interrogation method. The output of the fiber laser is frequency modulated with a fiber wrapped piezoelectric tube phase modulator, driven by a sinusoidal tone at $18.2 \mathrm{kHz}$. The modulator is used in reflection with a Faraday rotation mirror (FRM) to suppress polarization modulation. The modulated light is then injected into an imbalanced interferometer with a $1 \mathrm{~m}$ effective fiber path imbalance and a phase carrier signal is generated on the detector. The output of the detector is sampled by an A/D converted and the processing of the PGC signal is carried out in Labview. FRMs are used in the interferometer to ensure an optimum fringe visibility of greater than 0.9 . The section of fiber following the FL is a length of erbium doped fiber, which uses the remaining pump power to amplify the FL output to $10 \mathrm{~mW}$. The pump is injected into this section through a bi-pass wavelength division multiplexer (WDM) 
around the isolator (ISO). The interferometric phase shift, $\Delta \phi$, is related to the strain applied to the sensing fiber by the relation, $\Delta \phi=0.78 \cdot(4 \pi n l / \lambda) \cdot \Delta \varepsilon$, where $n$ is the effective refractive index of the optical fiber, $l$ is the sensing fiber length, $\lambda$ is the free space wavelength and $\Delta \varepsilon$ is the applied strain. The factor of 0.78 accounts for the elasto-optic effect [9].

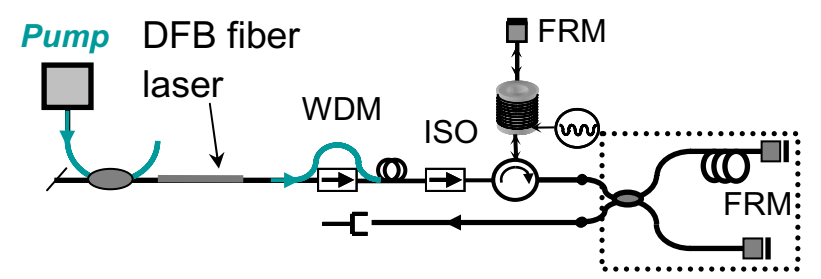

Figure 2: Interferometric sensor interrogated with DFB fiber laser formed with Michelson interferometer

\section{DFB FIBER LASER STRAIN SENSOR}

The fiber laser sensor configuration is shown in fig. 3. FL2 is used for these measurements and is also pumped by a grating stabilized pump diode at $980 \mathrm{~nm}$. The output of the FL is injected into one of three interferometer configurations. Configuration (i) is known as active-homodyne and uses a servo-loop to maintain the interferometer at its quadrature point. The phase signal is read from the feedback voltage applied to the PZT tube. The second configuration (ii) is known as the phase-generated carrier. Here, a sinusoidal tone is applied to the PZT, which generates a frequency carrier onto which the phase signals appear as modulation sidebands. The detected signal is then decoded in the same way as the interferometric sensor described above. Configuration (iii) is based on the 3-by-3 coupler and uses the inherent phase shift between the three outputs of the coupler to decode the phase signal. The interrogation approach described in [10] is used to decode the phase signal. The return loss of isolators (1) and (2) are 75 and $71 \mathrm{~dB}$ respectively, measured with a high resolution optical time domain reflectometer.

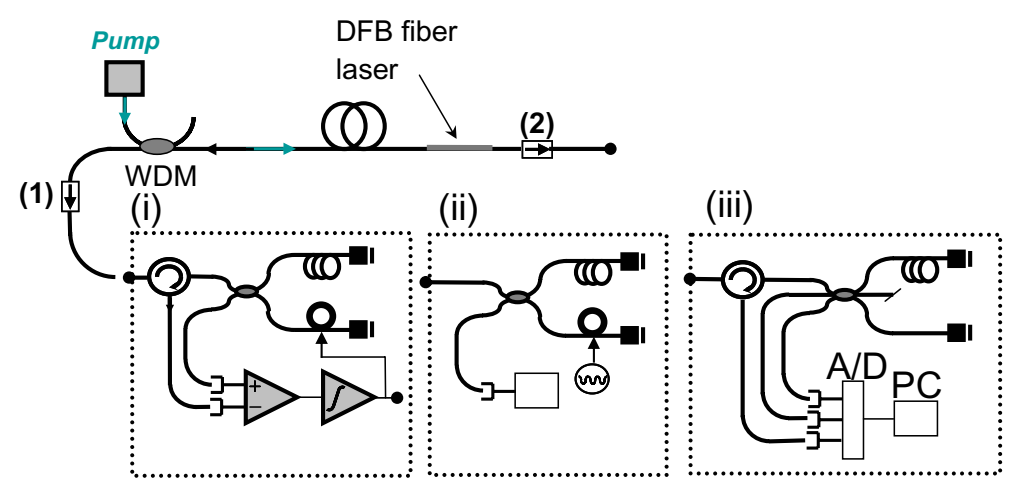

Figure 3: DFB fiber laser sensors decoded with (i) active-homodyne, (ii) phase generated carrier and (iii) $3 * 3$ interferometer

The interferometric phase shift is related to the strain applied to the fiber laser, $\Delta \varepsilon$, by the same relation as for the interferometric sensor. However, the length term, $l$, now corresponds to the imbalance in the decoding interferometer. This would be made large $(10-100 \mathrm{~m})$ to provide significant phase gain, such that the intrinsic laser noise can be made to dominate over all other noise sources. For the interrogation configurations above the fiber length imbalances are (i) $12.5 \mathrm{~m}$, (ii) $32 \mathrm{~m}$, and (iii) $42 \mathrm{~m}$, corresponding to phase gains of $0.78,2.01$ and $2.64 \mathrm{rad} / \mathrm{MHz}$ respectively

\section{STRAIN RESOLUTION COMPARISON AND DISCUSSION}

The strain resolution of the interferometric sensor is shown in fig. 4(a). A phase resolution of $2 \mu \mathrm{rad} / \mathrm{Hz}^{1 / 2} @ 2 \mathrm{kHz}$ is achieved, corresponding to a strain resolution of $2.10 \mathrm{p} \varepsilon / \mathrm{Hz}^{1 / 2}$ for a strain applied to $0.1 \mathrm{~m}$ of sensing fiber. Below $1 \mathrm{kHz}$, the phase resolution is limited by environmental noise. The laser frequency induced phase noise normalized to a fiber length imbalance of $1 \mathrm{~m}$ is also shown to fall about $10 \mathrm{~dB}$ below the sensor noise. Above $2 \mathrm{kHz}$ the sensor noise is limited by noise in the detector preamplifier. The strain resolution for the FLS using the three interrogation schemes described above is shown in fig. 4(ii), on the right-hand axis is shown the phase noise spectrum (normalized to an effective fiber length imbalance of $25 \mathrm{~m}$ ). The strain resolution achieved is $118 \mathrm{f} \varepsilon / \mathrm{Hz}^{1 / 2}$ at $2 \mathrm{kHz}$ corresponding to a phase resolution of 
$14 \mu \mathrm{rad} / \mathrm{Hz}^{1 / 2}$ for all three interrogation methods (apart from small variations in environmentally induced noise due to the measurements being taken at different times). In terms of minimum detectable length change, the MI achieves 210 $\mathrm{fm} / \mathrm{Hz}^{1 / 2}$ and the FLS achieves $0.76 \mathrm{fm} / \mathrm{Hz}^{1 / 2}$, an improvement factor of 275 . It follows that the fiber laser is capable of providing an increase in strain resolution by a factor of 18 , compared with a $10 \mathrm{~cm}$ length of fiber in an interferometric sensor. The strain resolution enhancement of the FLS is reduced as the length of interaction fiber in the interferometric sensor is increased. The strain resolution of the two sensors would be matched when the fiber interaction length in the interferometric sensor is $1.8 \mathrm{~m}$.

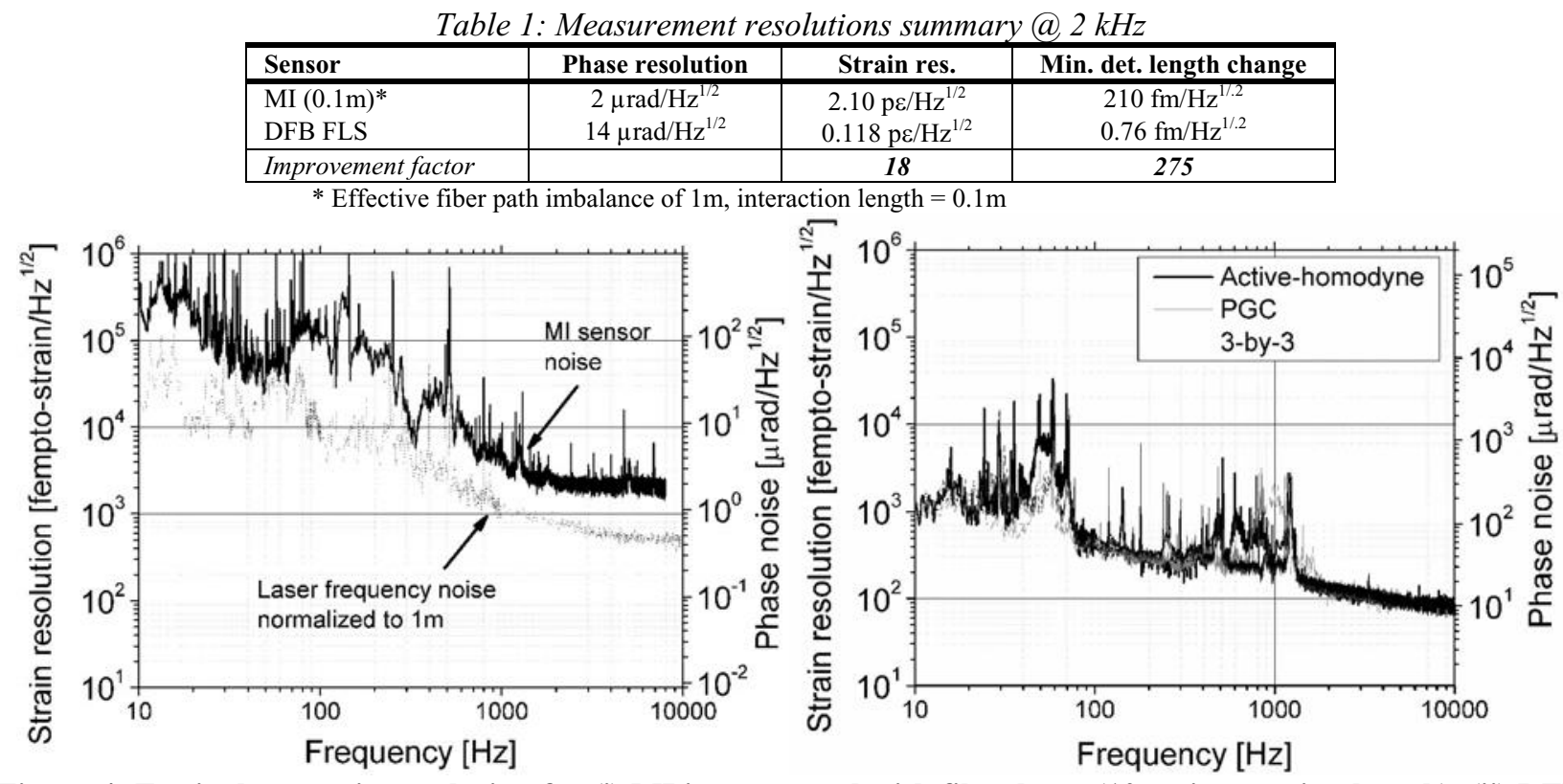

Figure 4: Equivalent strain resolution for (i) $M I$ interrogated with fiber laser $(10 \mathrm{~cm}$ interaction length), (ii) DFB fiber laser decoded with: active-homodyne (25m eff. fiber imbal); PGC (64m imbal); and 3*3 interferometer (84m imbal)

For applications where the interaction length is short $(<1 \mathrm{~m})$, the FLS provides a significant sensitivity benefit over the MI sensor (see for example [11]). The maximum sensitivity benefit is achieved if the interaction length is equal to the cavity length (approximately 5 and $6.5 \mathrm{~mm}$ for FL1 and 2 respectively). It should be noted that the frequency noise of the fiber laser deviates from the thermodynamic noise at frequencies below $\sim 1-2 \mathrm{kHz}$ depending on environmental conditions. This ' $1 / \mathrm{f}$ ' type noise of currently uncertain origin is often observed at lower frequencies. Thus, the benefit of the FLS may also be degraded at lower frequencies due to this excess noise.

\section{References:}

[1] G.A.Cranch, C.K.Kirkendall, K.Daley, S.Motley, A.Bautista, J.Salzano, P.J.Nash, J.Latchem, R.Crickmore, "Large-scale, remotely pumped and interrogated fiber-optic interferometric sensor array", IEEE Phot. Tech.Lett. 15(11), 1579-1581, (2003)

[2] K.J.Williams, A.Dandridge, A.D.Kersey, 1989 "Interferometric measurement of low-frequency phase noise characteristics of diode laser-pumped Nd-YAG ring laser" Elec. Lett, 25 (12), pp. 774-776

[3] J.T.Kringlebotn, J.L.Archambault, L.Reekie, D.N.Payne, 1994 " $\mathrm{Er}^{3+}: \mathrm{Yb}^{3+}$-codoped fiber distributed-feedback laser" Optics Letters 19 (24) 2101 2103

[4] D.J.Hill, P.J.Nash, D.A.Jackson, D.J.Webb, S.F.O’Neil, I.Bennion, L.Zhang, “A fibre laser hydrophone array” Fiber Optic Sensor Technology and Applications, SPIE 3860, Boston, MA, USA, 20-22 Sept. 1999, pp. 55-66

[5] D.Thingbø, E.Rønnekleiv, J.T.Kringlebotn "Intrinsic distributed feedback fibre laser high frequency hydrophone" Bragg Gratings, Photosensitivity, and Poling in Waveguides, 1999 Technical Digest

[6] S.Foster, A.Tikhomirov, M.Milnes, J.van Velzen, G Hardy, "A fibre laser hydrophone" Proc. 17 $7^{\text {th }}$ Int. Conf. Opt. Fibre Sensors, SPIE 5855, 23-27 May 2005, Bruges, Belgium, p. 627-629

[7] K.P.Koo, A.D.Kersey, "Bragg grating-based laser sensor systems with interferometric interrogation and wavelength division multiplexing ", $J$. Lightwave Technology, Vol. 13, no. 7, 1995

[8] K.H.Wanser, "Fundamental phase noise limit in optical fibers due to temperature fluctuations" Elec. Lett. 28(1), 53-54, (1992)

[9] C.D. Butter and G.B. Hocker, "Fiber optic strain gauge", Applied Optics, vol. 17, no. 18, pp. 2867-2869, (1978)

[10] A.D.Todd, G.A.Johnson, C.C.Chang, "Passive, light-intensity independent interferometric method for fibre Bragg grating interrogation" Elec. Lett. 35 (22), 1999, pp. 1971-1972

[11] G.A. Cranch, G.M.H.Flockhart, C.K.Kirkendall, "DFB fiber laser magnetic field sensor based on the Lorentz force" Proc. $18^{\text {th }}$ Int. Conf. Opt. Fib. Sensors, OSA Tech. Digest, ISBN 1-55752-817-9, Cancun, Mexico, Oct. 23-27, 2006 